\title{
Does monetary reward operantly enhance pain sensitivity over time? An experiment in healthy individuals
}

This article was published in the following Dove Press journal: Journal of Pain Research

\author{
Yukiko Shiro' \\ Tatsunori Ikemoto ${ }^{2}$ \\ Kazuhiro Hayashi ${ }^{3}$ \\ Young-Chang Arai ${ }^{4}$ \\ Masataka Deie ${ }^{2}$ \\ Takefumi Ueno ${ }^{5}$ \\ 'Department of Physical Therapy, \\ Faculty of Rehabilitation Sciences, \\ Nagoya Gakuin University, Aichi, \\ Japan; ${ }^{2}$ Department of Orthopaedics, \\ Aichi Medical University, Aichi, Japan; \\ ${ }^{3}$ Department of Rehabilitation, Aichi \\ Medical University, Aichi, Japan; \\ ${ }^{4}$ Institute of Physical Fitness, Sports \\ Medicine and Rehabilitation, School \\ of Medicine, Aichi Medical University, \\ Aichi, Japan; ${ }^{5}$ National Hospital \\ Organization Hizen Psychiatric \\ Center, Saga, Japan
}

Aim: Operant conditioning has long been believed to influence the pain experience through a psychological reward pathway. This study was formulated to test the hypothesis that pain sensitivity may be enhanced $>3$ months if a monetary reward works as a reinforcement.

Methods: Forty healthy subjects volunteered to participate in this study. The subjects repeatedly underwent pain testing via mechanical stimuli, and they rolled dice three (or six) times to gain money at the following five time points: baseline, three reinforcement sessions, and last session. The payoff was determined by roll of the dice. The subjects were instructed to roll the dice into a masked stand three times per session and informed that no one monitored the number of dice actually appeared. The subjects were also informed that they could roll the dice another three times when they reported strong pain during reinforcement sessions.

Results: The amount of individual payoff had significantly increased at last session compared with the values obtained at baseline; however, no changes were identified in terms of the pain ratings for mechanical stimuli during all sessions.

Conclusion: The results suggest that the psychological reward pathway does not always involve pain perception, and it is difficult to conclude whether pain sensitivity is operantly changed through the monetary reward in healthy individuals. Further investigation is required.

Keywords: pain sensitivity, chronic pain, behavioral manipulation, reinforcement

\section{Introduction}

Pain is a subjective experience that involves an emotional response. To date, many neuroimaging studies have focused on pain processing in the brain by classifying multiple dimensions. ${ }^{1}$ However, how pain is enhanced in the brain over the long term is not well understood. The sensory aspect of pain is commonly linked to the activation of nociceptors, nerves that signal mechanical, thermal, or chemical insults. ${ }^{2}$ Nevertheless, most pain clinicians worldwide believe that "Pain can occur without nociception". 3 The relationship between the activation of nociceptors and the pain experience is not concordant. ${ }^{4,5}$ Moreover, recent neuroimaging studies support this belief for patients with chronic pain. ${ }^{6,7}$ Madden et $\mathrm{al}^{3}$ reported that pain clinicians also believed that pain may be a classically conditioned response to a non-noxious stimulus, despite the scarcity of evidence to support this belief. One potential reason is that classical conditioning and operant conditioning have long been believed to influence the pain experience through the psychological reward pathway. ${ }^{8-12}$

Several procedures based on the classical conditioning or operant conditioning principle have been developed to investigate pain. For example, in laboratory stud-
Correspondence: Tatsunori lkemoto Department of Orthopaedics, Aichi Medical University, I-I Yazako Karimata, Nagakute, Aichi 480-I I95, Japan

$\mathrm{Tel}+81561623311$

Email tatsunon3I@gmail.com 
ies, authors have reported that verbal reinforcement altered the reports of pain sensitivity to noxious stimulus using the operant paradigm. ${ }^{10-12}$ Benedetti et al have recently reported that volunteer subjects who were informed that pain would be beneficial to muscle strengthening were more tolerant to ischemic pain than subjects who were only informed about the aversive nature of the task. They reported that the meaning of pain may change from negative to positive through the co-activation of the opioid and cannabinoid systems. ${ }^{13}$ However, these experiments were performed on a shortterm basis; thus, it remains unclear whether the classical conditioning or operant conditioning paradigms influence changes in the pain threshold in healthy subjects over the long term. Furthermore, previous studies could not estimate the relationship between the degree of reinforcement and the changes in pain sensitivity because reinforcers were often uncountable. In reinforcement learning studies, a monetary reward may often be used as a countable measurement. ${ }^{14}$ Several studies have indicated that seeking or receiving financial compensation following a motor vehicle collision leads to poorer recovery and worse pain. ${ }^{15,16}$ Zhou et a ${ }^{17}$ have reported that handling money diminished the physical pain of immersion in hot water, and moreover, a recent neuroimaging study has implied that monetary reward suppressed anterior insular activity, which involved with physical and social pain. ${ }^{18}$ These reports imply that financial gain over the long term may enhance pain sensitivities or lead to overt pain behavior and to chronic pain condition. However, there has been no evidence that repeated monetary reward could directly enhance pain sensitivities in normal adults.

Therefore, we aimed to investigate whether pain sensitivity changes over the long term using repeated monetary rewards in an experimental setting. In this study, we designed a paradigm in which the pain rating for a painful stimulus could increase the amount of monetary reward for healthy subjects. This experimental design enabled us to test the hypothesis that pain sensitivity and the amount of payoff, which could estimate the rate of lying, may be enhanced $>3$ months if a money reward works as a reinforcer for healthy individuals.

\section{Methods}

\section{Subjects}

Inclusion criteria were the adults who aged $>20$ years, were healthy, and had ability to consent autonomously. Exclusion criteria were as follows: any self-identified pain complaints, skin problem of the hand, a history of chronic pain or neurological problems such as peripheral neuropathy, and previous or current psychiatric diagnosis.
The sample size was estimated by the assumption of changes in the pain rating using a reinforcement protocol, which was reported by Jolliffe and Nicholas. ${ }^{12}$ They reported that $\sim 10 \mathrm{~mm}$ of a $100 \mathrm{~mm}$ visual analog scale (VAS) was different between reinforced subjects and nonreinforced subjects; therefore, we assumed that $10 \mathrm{~mm}$ changes in the mean VAS score with \pm 20 SD occurred over this paradigm. Based on this finding, at least 34 subjects were required to detect this assumption with a two-tailed $\alpha(0.05)$ and a power $\beta(0.8)$ in the priori analysis. Through subject recruitment with notice boards at colleges located in Aichi, Japan, 40 healthy subjects ( 25 men and 15 women) volunteered to participate in this study.

All subjects were final-year students in their colleges. The age of the subjects ranged from 21 to 23 years (mean $\pm \mathrm{SD}$; $22.45 \pm 1.01)$. The study received ethical approval from the Research Ethics Committee of the Aichi Medical University (2016-M027). All experiments were conducted from November 2016 to February 2017. After being informed of the purpose and protocol of the study, the subjects provided written informed consent prior to participating in the experiment.

\section{Study protocol}

The subjects repeatedly underwent experimental pain testing via mechanical stimuli, psychological questionnaire, and rolling dice. The subjects practiced a battery of measurements once before the experiment. The study was subsequently conducted at the following five time points: baseline, week 1 , week 2 , week 3 , and week 12 . Of these sessions, there were three reinforcement session for pain rating from week 1 to week 3 . These procedures are shown in Figure 1.

\section{Pain testing}

All experiments were conducted in a general room with the room temperature maintained within a range of $25^{\circ} \mathrm{C}-27^{\circ} \mathrm{C}$ and the humidity levels between $40 \%$ and $50 \%$. The experimental pain testing was based on the pain rating for mechanical stimuli.

A self-made von Frey monofilament (VFM) was prepared as a mechanical stimulating device. ${ }^{19}$ The diameters of the VFMs were $1.5 \mathrm{~mm}$ in all filaments, and the length of each monofilament (GCK-60 ${ }^{\circledR}$; Mitsubishi Reyon Co., Ltd., Tokyo, Japan) was adjusted to produce a different force (100 and $600 \mathrm{~g}$ ). The subjects sat in a fixed chair and placed their open right hands on the desk. Each painful stimulus was administered to two points of interdigital sites (second-third and fourth-fifth fingers) of the right hand for 5 seconds with 60 seconds interval for each measurement. A curtain was 

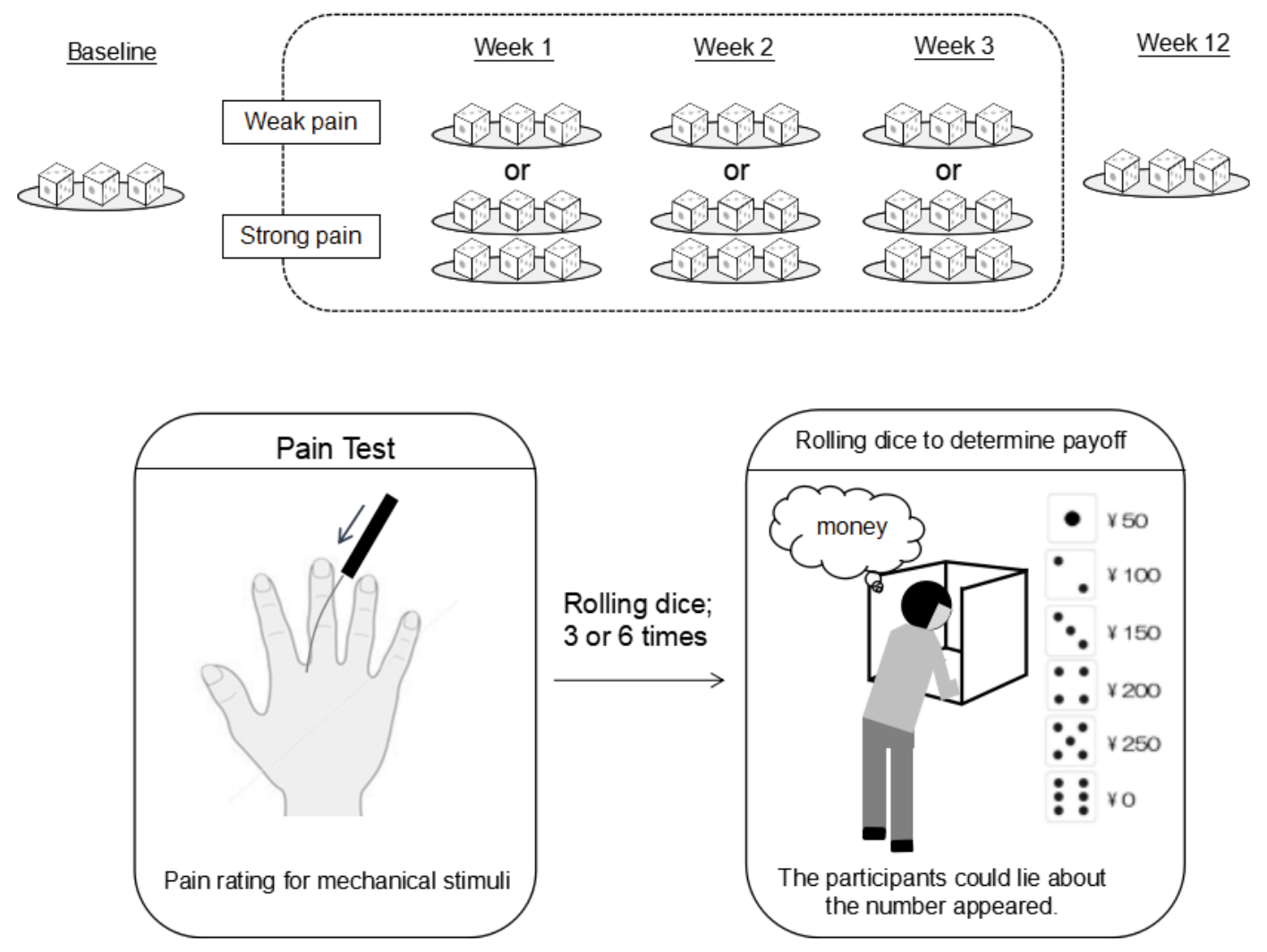

Figure I Protocol to test the hypothesis that pain sensitivity may be enhanced if a monetary reward is offered as reinforcement.

placed in front of the subjects to prevent them from viewing the stimulating filaments so as not to predict the specific types of VFM administered during the experiment. The subjects were only given information on the site of the stimulus. An experimenter $(\mathrm{KH})$, who applied mechanical stimuli to the subjects, was blinded to both any pain scores and payoff through all sessions. The pain VAS scores were reported every time to another experimenter (YS) whose position was at approximately $5 \mathrm{~m}$ distant from stimulating place at immediately after each stimulus. The pain VAS score was determined based on a $100 \mathrm{~mm}$ line labeled at the anchor points with "no pain" and "worst possible pain".

\section{Rolling dice procedure}

Figure 1 shows the protocol of this experiment. The rolling dice and payoff procedure were explained to the participants at the beginning of each session. To determine their individual payoff (= reward), the participants were instructed to roll the six-sided dice into a masked stand, which was not monitored by anyone. Prior to the experiments, all participants were informed that the payoff was decided by reporting the number of dice in 50 yen for numbers between 1 and 5 , namely 50 yen for 1,100 yen for 2, 150 yen for 3, 200 yen for 4, 250 yen for 5 , and 0 yen for 6 . The instructions also explicitly stated that the numbers of dice actually appeared would not be known to the experimenter. Thus, the subjects could easily be dishonest. Lying indicated reporting a different number than the number actually appeared. The participants reported the numbers for each roll of dice to the experimenter (YS) immediately after rolling procedure. It was impossible to detect lying at the individual level. The deceptive report was assumed only that the individual would receive a typically higher payoff than expected value by chance..$^{20,21}$

All participants were instructed to roll the dices three times at baseline and week 12. In contrast, from week 1 to week 3 , the subjects were only informed that "the subjects who perceived pain stronger than the mean score during the previous session can roll the dices six times and those who perceived pain less than that can roll the dices three times". The determination of "the mean score during the previous session" was kept a secret from the participants. Moreover, the secret determination of the number of times the dice was rolled represented the mean score ratings for the $100 \mathrm{~g} \mathrm{VFM}$ stimulus at the previous session. 
In this experiment, we calculated the theoretical value of payoff for each roll of dice because the numbers of rolling dice were different (three or six) by the participant from week 1 to week 3 . The payoff for each roll of dice was calculated as amount of payoff divided by the number of rolling dice for each participant at each session.

\section{Questionnaire}

We simultaneously examined the psychological aspect, which was catastrophizing in the subject every session, because it has been well established to be associated with pain and accordingly a potential covariate to pain sensitivities.

The Japanese version of the Pain Catastrophizing Scale (PCS) was used, which consists of 13 items that range from 0 to 52 and was composed of the following three subscales: rumination (eg, "I keep thinking about how much it hurts"), magnification (eg, "I wonder whether something serious may happen"), and helplessness (eg, "There is nothing I can do to reduce the intensity of the pain"). ${ }^{22,23}$

\section{Statistical analyses}

All continuous variables were presented as a mean and standard error (SE). The normality of the distribution was evaluated using the Shapiro-Wilk test for continuous variables. The pain VAS scores and amount of payoff at baseline and those at week 12 were compared using Student's $t$-test. One-way repeated-measures ANOVA were also used to compare the differences in these values across the sessions. The correlations of the pain VAS scores with the PCS scores and payoff for each roll of dice were analyzed using Pearson's test or Spearman's test, depending on the distribution of the data. All analyses were performed using the SPSS software (Version 24.0J; IBM Corporation, Armonk, NY, USA). Differences were considered statistically significant at a level of $P<0.05$.

\section{Results}

There were no significant changes in the pain VAS scores for the stimulus with $100 \mathrm{~g}$ VFM $(P=0.11)$ and $600 \mathrm{~g} \mathrm{VFM}$ $(P=0.67)$ between baseline and week 12 . In contrast, the amount of individual payoff was significantly increased at week 12 than at baseline $(P=0.02)$ (Figure 2).

Table 1 shows the pain VAS scores and payoff for each roll of dice at each session. One-way repeated-measures ANOVA showed that there were no significant differences in the pain VAS scores for the stimulus with $100 \mathrm{~g} \mathrm{VFM}$

Table I Pain VAS scores and payoff for each roll of dice at each session

\begin{tabular}{lllllll}
\hline & Baseline & Week I & Week 2 & Week 3 & Week I2 & Difference \\
\hline VAS-100 & $13.3(I .3)$ & $13.5(1.4)$ & $11.5(I .2)$ & $11.6(I .1)$ & $11.7(I .1)$ & $P=0.13$ \\
VAS-600 & $47.9(2.7)$ & $48.0(2.8)$ & $46.3(2.7)$ & $49.9(2.9)$ & $48.8(2.7)$ & $P=0.32$ \\
Payoff & $117.9(8.4)$ & $125.6(7.0)$ & $138.1(7.9)$ & $132.1(5.4)$ & $144.2(7.7)$ & $P=0.06$ \\
\hline
\end{tabular}

Notes: Values are shown as the mean (SE). One-way repeated-measures ANOVA were used to detect the differences in the values for each measure. VAS-100, VAS for $100 \mathrm{~g}$ stimulus; VAS-600, VAS for $600 \mathrm{~g}$ stimulus.

Abbreviations: SE, standard error; VAS, visual analog scale.

(A)

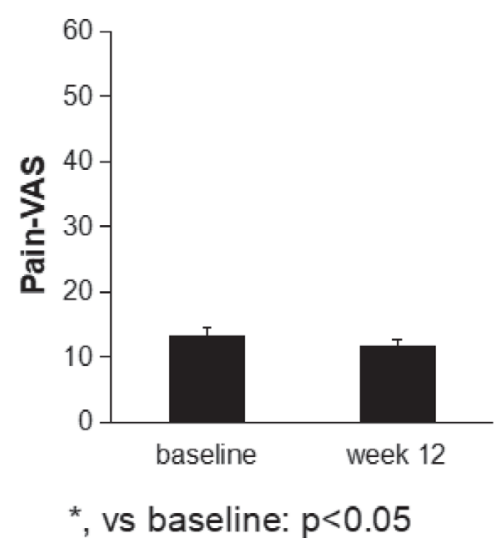

(B)
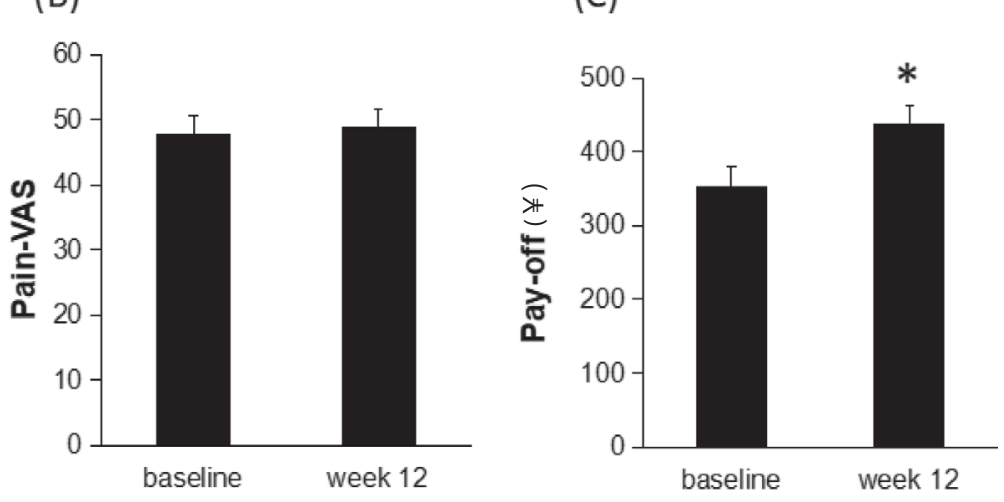

Figure 2 Pain VAS scores and amount of individual payoff.

Notes: (A) VFM of $100 \mathrm{~g}$, (B) VFM of $600 \mathrm{~g}$, and (C) amount of individual payoff. Pain VAS scores and the amount of payoff are shown as the mean (SE). *Compared to baseline: $P<0.05$.

Abbreviations: SE, standard error; VAS, visual analog scale; VFM, von Frey monofilament. 
and $600 \mathrm{~g} \mathrm{VFM}$ as well as the payoff for each rolling of dice through all sessions. However, the payoff for each rolling of dice was approximately chronologically increased through the sessions, which was close to significance $(F[4,39]=2.32$, $P=0.060$ ) (Table 1).

In terms of the psychological questionnaire, the PCS scores did not change at any session compared with those of the baseline. The pain VAS scores for both stimuli with $100 \mathrm{~g}$ VFM and $600 \mathrm{~g}$ VFM were significantly correlated with the PCS score, respectively (Table 2).

Figure 3 shows the distribution of reporting for the number of dice at each session. As expected, number 5 of dice was most frequently reported in total rolls. However, there were no relationships between the changes in pain sensitivities from baseline to week 12 and the total amount of payoff during the period from baseline to week 3 at the individual level (Table 3). Moreover, no relationship was identified between the mean score of the PCS and the amount of payoff.

\section{Discussion}

This study examined the hypothesis that pain sensitivity in healthy subjects could be enhanced $>3$ months if a monetary reward functioned as reinforcement in an experimental paradigm in which pain experience could increase the monetary reward. Although the amount of payoff significantly increased at week 12 than at baseline, in contrast to our expectation, pain sensitivities were not enhanced through sessions and there were no relationships between changes in pain sensitivities and the amount of payoff.

In this study, if the monetary reward successfully worked as reinforcement, a statistical change was expected to occur in the measurement of pain sensitivity. However, we could not identify changes in pain ratings for mechanical stimuli;

Table 2 PCS scores at each session and its correlation with each pain sensitivity

\begin{tabular}{|c|c|c|c|c|c|}
\hline & Baseline & Week I & Week 2 & Week 3 & Week I 2 \\
\hline \multirow[t]{2}{*}{ PCS } & $17.0(12.3)$ & $16.0(14.0)$ & $17.0(15.0)$ & $15.5(15.3)$ & I5.0 (I7.5) \\
\hline & VAS for $100 \mathrm{~g}$ stimulus & VAS for $600 \mathrm{~g}$ stimulus & & & \\
\hline PCS & $0.185^{\mathrm{a}}$ & $0.256^{b}$ & & & \\
\hline
\end{tabular}

Notes: Upper line: PCS scores are shown as mean (SE). Bottom line: correlation coefficient $(\rho)$ between $\mathrm{PCS}$ score and each pain sensitivity. ${ }^{\mathrm{a}}<<0.0 \mathrm{I}$ and ${ }^{\mathrm{b} P}<0.00 \mathrm{I}$. Abbreviations: PCS, Pain Catastrophizing Scale; SE, standard error; VAS, visual analog scale.

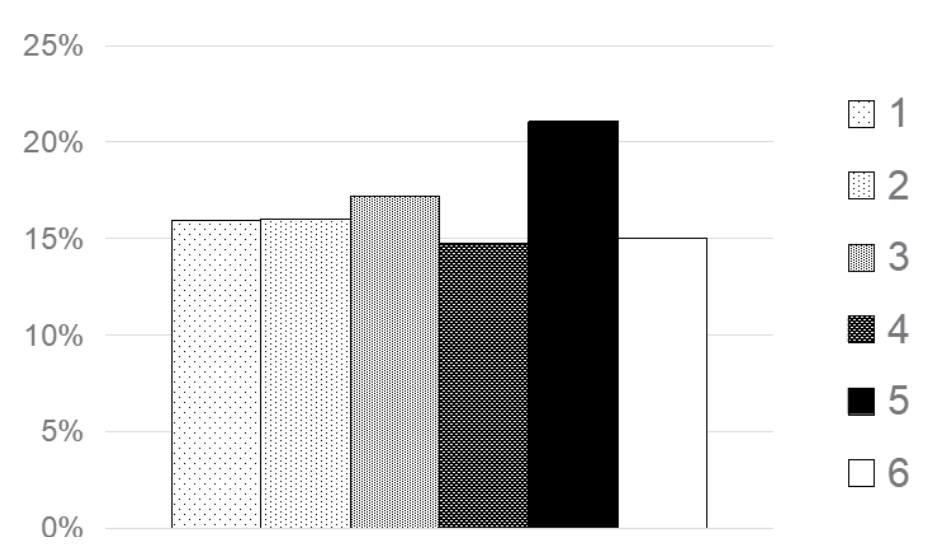

Figure 3 Distribution of reported number of dice in total rolls.

Table 3 Correlation between payoff and change in pain sensitivity

\begin{tabular}{ll}
\hline & Changes in pain sensitivities between baseline and week I2 \\
\cline { 2 - 2 } Total amount of payoff (up to week 3 ) & -0.131 \\
\hline
\end{tabular}

Notes: Value: a correlation coefficient. Statistical analysis was conducted using Pearson's correlation or Spearman's correlation, depending on the distribution of data. Abbreviation: VAS, visual analog scale. 
thus, it was difficult to conclude whether the pain sensitivity was affected by the money reward in this paradigm. The factors of developing chronic pain include not only peripheral tissue vulnerability but also central pain modulation with neuroplasticity. ${ }^{24,25}$ Several articles have reported that the pain sensitivity was modulated by positive verbal or monetary reinforcement. ${ }^{10,12,26}$ Moreover, Fordyce ${ }^{8}$ indicated financial gain as a potential reinforcer for chronic pain behavior. Therefore, our primary speculation was that pain ratings for noxious stimuli would chronologically increase to gain a preferable outcome because they could roll the dice more times when they showed more intense pain. Contrary to our expectations, the results did not indicate enhancements of pain sensitivities. In the complex biopsychosocial nature of chronic pain after whiplash injury, many believe that compensation does more harm than good, but there is no clear evidence to support an idea that compensation and its related processes lead to worse health. ${ }^{27}$ Implication of this study is that psychological reward pathway does not always involved with pain perception in normal adults, although this relationship may depend on socioeconomic background of subject. The lack of socioeconomic background of the subjects is one of the limitations in this study.

We need to discuss an inconsistency of our results with previous reports. ${ }^{10,12,26}$ The potential explanations in terms of the inconsistency are discussed here. First, the magnitude of the reinforcer may be insufficient to affect the pain perception, namely, the frequency and degree of reinforcement. Some authors have suggested that there were an extrinsic reinforcement and an intrinsic reinforcement in the operant paradigm. ${ }^{28,29}$ In this study, monetary reinforcement as an extrinsic reinforcement, which meant a modality external to the nociceptive pathway was only administered three times for 3 months. Therefore, this frequency may be insufficient to affect pain perception. We also speculated that the amount of monetary reward may not reach out to affect pain reports; in other words, several times more money than the reward in this study may induce overt pain reports or change pain sensitivities. Second, this paradigm required a long interval (1 week) to obtain the next reinforcement compared with previous experiments (eg, long-term sensitization vs short-term sensitization), which was insufficient to affect the pain perception. Third, although the reinforcer in previous studies was automatically given in accordance with a protocol, this study enabled the subjects to get more money by more deceptive reports of the number of dice (eg, 555 ) as well as by reporting more intense pain. The amount of payoff appeared to be increased in the latter ses- sions; however, we did not identify relationships between the amount of payoff and pain experience. It was speculated that there were not many lies when they reported the number of dice in this study. Since the deception estimated by the distribution of reporting the number of dice in this study appeared to be substantially less than that in previous studies, ${ }^{20,21}$ there may be differences across races or cultures.

In addition to the operant modulation, we investigated the psychological factor of the participants as one potential covariate associated with pain sensitivity. As expected, the pain ratings for both mechanical painful stimuli positively correlated with the PCS scores; however, no relationship was found between PCS score and individual payoff. Furthermore, this study was only conducted in healthy volunteers and not patients with chronic pain. This operant paradigm by monetary reward for long-term pain sensitization may be unsuitable for healthy subjects. Further investigation is required to detect the decisive reinforcer that may enhance the pain sensitivity over the long term even in healthy subjects.

\section{Conclusion}

This study examined the hypothesis that pain sensitivity may be enhanced $>3$ months if a money reward works as the reinforcer for healthy individuals. Although the amount of payoff was significantly increased at week 12 than at baseline, in contrast to our expectation, pain sensitivities were not enhanced through sessions, and there were no relationships between changes in pain sensitivities and the amount of payoff.

Further investigation is required to detect the decisive reinforcer that may enhance pain sensitivity over the long term even in healthy subjects.

\section{Author contributions}

YS, TI, and TU contributed to the study concept and design. All authors contributed toward data analysis, drafting and critically revising the paper and agree to be accountable for all aspects of the work.

\section{Disclosure}

The authors report no conflicts of interest in this work.

\section{References}

1. Wager TD, Atlas LY, Botvinick MM, et al. Pain in the ACC? Proc Natl Acad Sci U S A. 2016;113(18):E2474-E2475.

2. Gold MS, Gebhart GF. Nociceptor sensitization in pain pathogenesis. Nat Med. 2010;16(11):1248-1257.

3. Madden VJ, Moseley GL. Do clinicians think that pain can be a classically conditioned response to a non-noxious stimulus? Man Ther. 2016;22:165-173. 
4. Sprenger C, Eippert F, Finsterbusch J, Bingel U, Rose M, Büchel C. Attention modulates spinal cord responses to pain. Curr Biol. 2012;22(11):1019-1022.

5. Villemure C, Bushnell MC. Mood influences supraspinal pain processing separately from attention. J Neurosci. 2009;29(3):705-715.

6. Lee MC, Tracey I. Unravelling the mystery of pain, suffering, and relief with brain imaging. Curr Pain Headache Rep. 2010;14(2):124-131.

7. Apkarian AV, Hashmi JA, Baliki MN. Pain and the brain: specificity and plasticity of the brain in clinical chronic pain. Pain. 2011;152(3 Suppl):S49-S64.

8. Fordyce WE. Behavioural Methods for Chronic Pain and Illness. Mosby: St Louis, MO; 1976

9. Li JX, Jx L. The application of conditioning paradigms in the measurement of pain. Eur J Pharmacol. 2013;716(1-3):158-168.

10. Linton SJ, Götestam KG. Controlling pain reports through operant conditioning: a laboratory demonstration. Percept Mot Skills. 1985;60(2):427-437.

11. Flor $\mathrm{H}$, Knost B, Birbaumer N. The role of operant conditioning in chronic pain: an experimental investigation. Pain. 2002;95(1-2):111-118.

12. Jolliffe $C D$, Nicholas MK. Verbally reinforcing pain reports: an experimental test of the operant model of chronic pain. Pain. 2004;107(1-2):167-175.

13. Benedetti F, Thoen W, Blanchard C, Vighetti S, Arduino C. Pain as a reward: changing the meaning of pain from negative to positive coactivates opioid and cannabinoid systems. Pain. 2013;154(3):361-367.

14. Lin A, Adolphs R, Rangel A. Social and monetary reward learning engage overlapping neural substrates. Soc CognAffect Neurosci. 2012;7(3):274-281.

15. Harris I, Mulford J, Solomon M, van Gelder JM, Young J. Association between compensation status and outcome after surgery: a metaanalysis. JAMA. 2005;293(13):1644-1652.

16. Murgatroyd DF, Cameron ID, Harris IA. Understanding the effect of compensation on recovery from severe motor vehicle crash injuries: a qualitative study. Inj Prev. 2011;17(4):222-227.
17. Zhou X, Vohs KD, Baumeister RF. The symbolic power of money: reminders of money alter social distress and physical pain. Psychol Sci. 2009;20(6):700-706.

18. Cristofori I, Harquel S, Isnard J, Mauguière F, Sirigu A. Monetary reward suppresses anterior insula activity during social pain. Soc Cogn Affect Neurosci. 2015;10(12):1668-1676.

19. Hayashi K, Ikemoto T, Ueno T, et al. Regional differences of repeatability on visual analogue scale with experimental mechanical pain stimuli. Neurosci Lett. 2015;585:67-71.

20. Fischbacher U, Heusi F. Lies in Disguise: An Experimental Study on Cheating. Thurgau Research Paper Series. 2008;40:1-20.

21. Wibral M, Dohmen T, Klingmüller D, Weber B, Falk A. Testosterone administration reduces lying in men. PLoS One. 2012;7(10):e46774.

22. Sullivan MJL, Bishop SR, Pivik J. The Pain Catastrophizing Scale: Development and validation. Psychol Assess. 1995;7(4):524-532.

23. Matsuoka H, Sakano Y. Assessment of cognitive aspect of pain:development, reliability, and validation of Japanese version of PainCatastrophizing Scale. Jpn J Psychosom Med. 2007;47:95-102.

24. Apkarian AV, Baliki MN, Farmer MA. Predicting transition to chronic pain. Curr Opin Neurol. 2013;26(4):360-367.

25. Denk F, Mcmahon SB, Tracey I. Pain vulnerability: a neurobiological perspective. Nat Neurosci. 2014;17(2):192-200.

26. Baker SL, Kirsch I. Cognitive mediators of pain perception and tolerance. J Pers Soc Psychol. 1991;61(3):504-510.

27. Spearing NM, Connelly LB, Gargett S, Sterling M. Does injury compensation lead to worse health after whiplash? A systematic review. Pain. 2012;153(6):1274-1282.

28. Hölzl R, Kleinböhl D, Huse E. Implicit operant learning of pain sensitization. Pain. 2005;115(1-2):12-20.

29. Becker S, Kleinböhl D, Klossika I, Hölzl R. Operant conditioning of enhanced pain sensitivity by heat-pain titration. Pain. 2008;140(1):104-114.
Journal of Pain Research

\section{Publish your work in this journal}

The Journal of Pain Research is an international, peer reviewed, open access, online journal that welcomes laboratory and clinical findings in the fields of pain research and the prevention and management of pain. Original research, reviews, symposium reports, hypothesis formation and commentaries are all considered for publication.

\section{Dovepress}

The manuscript management system is completely online and includes a very quick and fair peer-review system, which is all easy to use. Visit http://www.dovepress.com/testimonials.php to read real quotes from published authors. 